\title{
Tecnología e ideas usadas para el análisis espacial de paisajes arqueológicos en la Argentina
}

(4) María J. Figuerero Torres*

"While technology is important, it's what we do with it that truly matters."

Muhammad Yunus (2012)

En este Dossier, M. E. De Feo, E. Moreno y L. Magnin han reunido un conjunto de trabajos que muestran lo que los arqueólogos podemos hacer con la tecnología apropiada cuando esta se encuentra a nuestra disposición. Bajo el título de "Herramientas analíticas para el estudio del paisaje en la Arqueología Argentina. Cruzando fronteras y tiempos" han reunido ocho análisis arqueológicos de sitios y regiones de la Argentina. En lo que sigue, daré mi visión de lo que han podido hacer estos autores en cada uno de sus estudios del paisaje. Espero que esto sea una guía para descubrir las ideas que, gracias a la tecnología del análisis espacial, lograron cruzar esas fronteras y tiempos.

Podemos hacer una síntesis de los rasgos sobresalientes de los trabajos de este volumen. Para comenzar, se agrupan en solo dos grandes regiones: el Noroeste Argentino (NOA) y sur de Patagonia y Tierra del Fuego, Argentina. Temporalmente hay algo de coincidencia en los registros cronológicos analizados entre algunos de los trabajos del NOA, como así también entre algunos de los del extremo sur. Por último, la mayoría emplea a los SIG (Sistemas de Información Geográfica) como la herramienta base de su análisis. Pero esta caracterización es engañosamente simplista y no hace justicia a la diversidad de enfoques, la innovación tecnológica empleada, los variados tipos de análisis y el grado de complejidad de las bases de datos analizadas, junto con los resultados alcanzados consecuentemente en cada caso. Es justamente esta diversidad la que hace tan atrayente al conjunto de los trabajos que han convocado los editores del Dossier y amerita este breve análisis previo.

\section{Los trabajos}

En los comentarios que siguen, examino los trabajos en función de la etapa de investigación sobre la cual arrojan luz los resultados presentados. Esto es, si contribuyen al relevamiento inicial de un sitio o región, si es para interpretar los patrones de distribución de hallazgos o redirigir el trabajo de prospección o si el objetivo fue ahondar en la comprensión de diferentes porciones de un registro ya extensamente analizado.

Hay un solo trabajo que refiere a la etapa inicial de descubrimiento de la investigación arqueológica. A la vez, constituye un hito metodológico para la arqueología argentina y tiene en este Dossier su primera publicación. Me refiero al trabajo de Moralejo, Gobbo,

* Instituto de Arqueología, Facultad de Filosofía y Letras, Universidad de Buenos Aires (UBA). 25 de mayo 217, $3^{\circ}$ piso (CP C1002ABE) Ciudad Autónoma de Buenos Aires, Argentina. E-mail: mjofiguerero@yahoo.com.ar 
Del Cogliano y Pinto, en el que exponen el empleo de la tecnología LIDAR (Light Detection and Ranging) para el relevamiento del sitio inka de El Shincal de Quimivil en Catamarca. No es que faltara información sobre el sitio, que cuenta con investigaciones que remontan a comienzos del siglo XX y que se potenciaron con el trabajo liderado por Rodolfo Raffino a partir de la década del 1980. Lo analizado hasta el momento indica que El Shincal es uno de los asentamientos inka más importantes en la Argentina, emplazado en un nodo de la red del camino inka principal o Qhapaq Ñan. No obstante, sus dimensiones, la aparente diversidad de estructuras en lo aún no trabajado, el sedimento que ha enterrado al sitio y la abundante vegetación potencialmente esconden aún más información sobre el significado de este asentamiento y su relación con la región circundante. Esto fue el incentivo para lograr implementar una tecnología de alta precisión, que brinda mediciones en $3 \mathrm{D}$, es no intrusiva y tiene la capacidad de abarcar grandes áreas para relevar este sitio tan complejo. Los autores presentan el diseño de investigación, cuyo esfuerzo crucial, en realidad, fue elaborar una estrategia de colaboración para hacerlo efectivo y cubrir los muy elevados costos de ejecución. Esto conllevó la compatibilización de objetivos entre las partes involucradas, implementar la logística desplegada en el campo, la recolección y procesamiento de la información y hasta el control posterior en el terreno. Los resultados iniciales no solo son relevantes para ampliar lo discutido sobre este centro administrativo inka y la red de caminos, sino también para comprender los procesos erosivos geomorfológicos que afectan el área y poder consolidar una versión virtual del patrimonio del lugar. Es evidente que los resultados previstos sobre la base de lo realizado representan una mera fracción del potencial que tiene la enorme cantidad de información recolectada. Este caso presentado permite evaluar lo que implican los costos altos a corto plazo versus los resultados potenciales a largo plazo. La implementación de esta metodología en otras regiones de la Argentina dependerá principalmente de buscar la participación y beneficio simultáneo para otros organismos y partes interesadas para solventar los gastos involucrados.

Los tres análisis localizados en el extremo sur de Patagonia y Tierra del Fuego los incluí en el grupo de trabajos que se centran sobre la interpretación de las distribuciones arqueológicas. No obstante, el acercamiento y los materiales empleados en cada análisis son muy diversos. La cuenca del río Santa Cruz, en el sur de la Patagonia argentina, es actualmente foco de atención a raíz de la construcción de dos megarepresas para el aprovechamiento hidroeléctrico del río, a la que se opone una gran resistencia liderada por una coalición de ambientalistas. A las investigaciones arqueológicas en la cuenca media, iniciadas en los últimos años, se está sumando ahora la información proveniente del trabajo de impacto y salvataje que se está llevando adelante. El análisis de Gilio se nutre de ello para modelar los caminos óptimos que pudieron unir puntos de inicio y fin localizados en márgenes opuestas del río. La relevancia de estos puntos es que están conformados por sitios arqueológicos con cronología y material contextual ya analizado, que han servido para ver diferencias en la composición de los contextos en ambas márgenes del río. Los caminos óptimos que surgen del modelo SIG de Gilio son importantes para marcar posibles vías o pasos para sortear la barrera que potencialmente representa el río. La construcción del modelo se basa en criterios físicos de tránsito por el terreno que después Gilio compara con las distribuciones arqueológicas conocidas hasta el momento. Este es un análisis inicial y exploratorio y la autora misma señala que el desarrollo futuro se beneficiará de criterios adicionales, como ser datos sobre el modo de moverse en el terreno y de los que surgen de los mismos contextos arqueológicos. En principio, está el potencial del modelo para brindar nuevos parámetros al interpretar el registro ya localizado, como también para programar el trabajo de prospección de los sectores a relevar en el poco tiempo que aún resta. Tiene también su valor para rescatar potenciales paisajes culturales, al modelar un territorio extenso que está próximo a desaparecer en forma irremediable inundado por los espejos de agua de las represas proyectadas. 
En la costa Atlántica de Tierra del Fuego el trabajo de Negré, Briz-i Godino, Pal y Álvarez construye un modelo con base en SIG para ver la dependencia entre determinadas variables ambientales con la ubicación de los sitios arqueológicos y su consiguiente agrupación. El sector analizado en este caso es una porción de unos $40 \mathrm{~km}$ a lo largo de la costa en una zona de ecotono entre la estepa y el bosque fueguino. Aquí, el marco ambiental incluyó a los recursos naturales biológicos (fauna y flora) así como también la información geomorfológica de las variadas formas que componen el terreno. A diferencia del trabajo de Gilio, aquí se empleó el conocimiento de la ubicación de cientos de sitios y distribuciones relevados en la última década en el sector para los que se presume una cronología del Holoceno tardío reciente. Pero, a partir de este patrón, se suma el análisis del grado de agrupación de los sitios entre sí para aportar mayores precisiones sobre el posible sistema de asentamiento. Sobre la base de la accesibilidad de recursos entonces, los autores han podido ver la conformación de áreas de concentración de múltiples ocupaciones sucesivas en el tiempo. Lograron así rever variables, muchas veces asumidas como de mayor peso que intervienen en la localización de los asentamientos costeros y tener mejor base para comprender las ocupaciones reiteradas en un sector dado. El cuidadoso análisis estadístico que sostiene este modelo es una excelente herramienta para guiar trabajos a futuro en el sector y un punto de comparación con otras porciones costeras de la Isla Grande.

El tercer trabajo dentro de este grupo se diferencia por estar centrado en un hecho emblemático de la historia reciente de la Patagonia. El conocimiento de las Huelgas Obreras de los peones rurales de 1920-1921, ahora también llamada "La Patagonia rebelde", deriva en gran parte de la memoria oral y de documentos históricos rescatados mucho después de ocurrido el hecho, con inevitables contradicciones y omisiones entre ambos tipos de fuentes para algunos incidentes. El aporte de Zubimendi es analizar los sucesos de la huelga de 1921 en la porción norte de la provincia de Santa Cruz. Para esto se basó no solo en documentación conocida sino que también pudo agregar fuentes documentales nuevas. Esta base de archivo se vio ampliada por la contrastación con los restos materiales de los sucesos identificados en el terreno. El trabajo de fijar los sucesos en el tiempo y espacio con el uso de un SIG, no solo fue un trabajo de reconstrucción sino además de recuperación de otros incidentes aislados olvidados. Este análisis tiene valor para un posicionamiento más fundado de los eventos que componen la Huelga Obrera en las zonas rurales más alejadas del norte de la provincia y una revalorización de la memoria de los hechos aún de gran significado emotivo para las generaciones presentes. En esto último, la original iniciativa de Zubimendi se agrega a recientes revisiones de incidentes históricos cercanos en el tiempo, como ser las muertes masivas de obreros en Alto Pisagua, enterrados en la Isla de los muertos en el río Baker, Aysén, a comienzos del siglo XX, que han sido revalorizados mediante el análisis arqueológico y de antropología histórica (Osorio Pefaur, 2015).

El último grupo de trabajos reúne análisis de lugares o áreas de investigación con muchos años de trabajo y con resultados de la información contextual de los sitios analizados o de otros en la región. Los análisis fueron llevadas a cabo en múltiples escalas y son a la vez muy complejos dada la cantidad de información que pudieron incluir los autores en forma simultánea.

En el trabajo de Izaquirre y Ferrari tenemos un análisis muy novedoso de la arqueología de los sentidos llevado a cabo para una estructura en Las Pailas, Salta, un sitio arqueológico del Período Tardío del NOA. El trabajo previo en este sitio data de mediados del siglo XX y, junto con trabajos más recientes, muestran que Las Pailas es un poblado conglomerado con áreas diferenciadas, incluyendo una zona residencial con sectores de estructuras articuladas, asociadas a vías de circulación y un número de grandes espacios públicos a lo largo del sitio. Los autores seleccionaron uno de estos espacios públicos para el análisis, junto con el sendero que lo rodea y los recintos adyacentes. La recolección de la información necesaria y el procesamiento 
son sumamente complejos y consisten en generar los parámetros de visibilidad para las estructuras y luego de lo sonoro para poder evaluar su co-ocurrencia. Implicó además un trabajo original de reconstrucción de las condiciones físicas que pudieron existir para influir sobre la dispersión sonora, como ser el material de las paredes y su textura así como la potencia de la voz. El análisis en sí de las diferentes formas de percepción fue asimismo cuidadoso, contraponiendo lo que se veía y escuchaba en diferentes posicionamientos y trayectorias, tanto dentro como fuera del espacio público. El resultado es una innovadora forma de apreciar la interacción social que articuló estos espacios y que no podría derivarse solo del plano bidimensional o visual exclusivamente. Constituye, por lo tanto, un original acercamiento para aumentar la comprensión acerca de la organización social jerárquica de Las Pailas y de otros poblados semejantes para el Período Tardío en el NOA.

La Quebrada de Incahuasi en Salta es otra área que cuenta con muchos antecedentes de investigación sobre sociedades pastoriles y agrícolas del NOA. Para este volumen De Feo analiza el arte grabado que se halla en cientos de bloques dispersos a lo largo de cauces activos y secos, delimitando vegas, o cercanos a recintos, en el paisaje de la quebrada que conecta diferentes zonas altitudinales. La ocupación evidenciada por los recintos y materiales abarca desde el Período Formativo hasta el Período Tardío. El análisis espacial de los bloques con arte se orientó a conocer la persistencia de su uso por los sucesivos ocupantes y cómo fueron percibidos por ellos al transitar el mismo paisaje. Lo atractivo del análisis es la claridad con la que las medidas se relacionan con los comportamientos que se quieren medir. Por eso los resultados van más allá de la distribución cronológica de los petroglifos y habilitan a De Feo a inferir la forma en que fue utilizado el camino por los pastores y cómo los bloques grabados previamente fueron luego respetados, integrándolos a los desplazamientos en el paisaje. Los cambios en la intensidad y en la distribución o visibilización de los motivos también la habilitan a discutir la relación entre el pastoreo y caravaneo en la quebrada como así también la expresión local de los cambios en la organización social propuestos para estos períodos a nivel regional en el NOA.

Las escalas de análisis empleadas en el trabajo de Cahiza, Sabatini e Iniesta son varias y se centran sobre los restos arqueológicos en tres cuencas del piedemonte de la Sierra de Velazco, La Rioja. Aquí el estudio también se benefició del amplio trabajo previo sobre estructuras diversas y materiales variados, en una superficie que supera los $100 \mathrm{~km}^{2}$. La base de datos es muy rica y apropiada para inferir, a través del empleo de tecnologías SIG, la asociación espacial de los asentamientos residenciales y los espacios productivos con cada una de las cuencas aluviales. Las otras medidas usadas fueron las distancias entre estructuras que permitieron definir la cercanía entre espacios residenciales y productivos, que sirvieron de base para inferir el grado de planificación existente. Así como el rango de tamaño de las estructuras fue el pie para ver las diferencias en la jerarquización dentro y entre cuencas. Esta breve descripción soslaya el cuidadoso análisis de los datos relevados y los patrones que van construyendo los autores. El resultado muestra diferencias muy informativas sobre posibles acciones grupales o individuales en la elección de los lugares y en las prácticas agrícolas en las tres cuencas, que podría ser resultado de una planificación familiar más que comunitaria. Una vez lograda esta sólida base de patrones espaciales de estructuras y materiales, los autores recién proponen tendencias temporales dentro del bloque temporal analizado, 300 a 900 años d. C., tanto para la organización de las estructuras relevadas como de los esquemas sociales acompañantes. Este minucioso análisis presenta entonces una matizada interpretación de un componente arqueológico importante que permite relacionarlo con el Formativo Tardío en otras regiones del NOA y brindar una base fundada para evaluar los procesos sociales que ocurren en este transcurso.

En su trabajo sobre la Quebrada de Antofalla, Catamarca, Moreno resume un gran cuerpo de información fruto de muchos años de trabajo y de una enorme diversidad en 
la naturaleza de evidencia recolectada. En forma acorde, el análisis en un SIG se manejó en varias escalas, que van desde lo puntual del artefacto hasta las relaciones sociales establecidas a distancia a través de los materiales empleados como recursos. Cada una de estas escalas de análisis se combina para delimitar las prácticas relacionadas con la agricultura, el pastoreo y la caza, que rara vez se pueden hacer con cuerpos comparables de evidencia arqueológica. El análisis marca espacios sobre la base de la presencia de estructuras, materiales superficiales y aquellos provenientes de excavaciones. No solo lo artefactual y constructivo fue considerado sino que se incorporó el uso de las materias primas arqueológicas empleadas, como ser la obsidiana de fuentes identificadas y lo modelado sobre la base de saberes actuales. Movimientos e interacciones dan como resultado una dinámica en las variadas prácticas que Moreno evalúa en su profundidad $\mathrm{y}$ continuidad para un paisaje que concibe como campesino.

\section{Acercamientos conceptuales y redes de referencia}

Resta en este comentario destacar otro aspecto de la gran diversidad de los trabajos, que complementa lo dicho sobre algunos rasgos comunes. Donde realmente se aprecia esta diversidad es en el uso de los conceptos teórico-metodológicos que emplean los autores y en la red de referentes con los que se relacionan.

Al usar la palabra territorio como algo conceptual hay una base común entre los autores de anclarlo en el ámbito físico del terreno. Algunos autores no enfatizan su uso, como Izaguirre y Ferrari que no incluyen la expresión en su trabajo pero, dentro de la arqueología de los sentidos, la parte sensorial se basa sobre la percepción que, en sí, comprende a la porción física del ambiente. En forma también amplia, Moralejo et al. sitúan al territorio junto con la información geográfica general sobre los lugares y el espacio. En cambio, otros autores lo aclaran en forma explícita porque es parte nodal de su análisis. Este es el caso de Negré et al., para quienes el territorio es el espacio físico contenedor de las relaciones de producción y reproducción humana. Para ello, remiten a conceptos desarrollados por geógrafos europeos como referentes para su definición. De modo semejante, Gilio también emplea al concepto de territorio como un espacio físico dado pero en su planteo los conceptos referentes fueron generados por arqueólogos estadounidenses. En el caso de Cahiza et al., estos autores no usan explícitamente el término pero podemos inferir que claramente distinguen un aspecto físico al considerar los componentes ecológicos y los recursos donde tienen lugar las interacciones de la sociedad con el ambiente. En cambio De Feo, sí aclara que su análisis incluye los atributos ecológicos del paisaje, como marco de relaciones y circuitos de movilidad, y en el aprovechamiento de recursos y manejo de camélidos que, en conjunto, le dan sustancia al significado de lugares. Mientras que Moreno, tiende a destacar el aspecto más social del territorio, al referir a un espacio ocupado y bajo control que se define por las prácticas de explotación que se realizan, sean estas agrícolas, pastoriles o cinegéticas. El trabajo de Zubimendi, tal vez por estar situado en el siglo XX, no precisa del término pero sí distingue un medio físico en el cual se insertan acciones humanas y que es clasificable por su uso con respecto a otros sectores. De modo que vemos que todos los autores incluyen el uso del concepto de territorio, con mayor o menor énfasis, como un aspecto físico, pero que está mediado por las relaciones humanas de transformación de los recursos que en ella se dan. No todos los trabajos explicitan los referentes del uso que le dan al concepto de territorio y, cuando esto sucede, son fuentes diversas en cuanto a corrientes teóricas y origen disciplinar.

Una exploración más interesante acerca de la diversidad entre los trabajos, ocurre cuando examinamos el uso conceptual que tiene el término espacio en relación con el comportamiento humano. Aquí incluyo además la manera en que los trabajos incorporan la noción de tiempo, el par natural del espacio como concepto. 
En el trabajo de Negré et al., los autores conciben al espacio en términos del agente espacial humano que se apropia del espacio y para ello se basan nuevamente en los esquemas conceptuales de geógrafos europeos. La diacronía no es el objeto de este análisis, pero el patrón resultante al analizar la agrupación de los sitios es explicado como el producto de la reocupación de lugares que implica la duración, entre otros elementos, y refieren para ello a conceptos de arqueólogos de la Argentina, Estados Unidos y Europa. Para definir a los caminos, Gilio los considera como articuladores de los espacios que contienen a la movilidad y circulación humana. Pero, al considerarlos también construcciones históricas, incorpora la noción de tiempo como concepto operativo que, en su análisis, por el momento solo está empleado para definir la dirección del trazado de los caminos modelados. En todo esto Gilio remite a conceptos elaborados tanto por otras arqueólogas de la Argentina y Sudamérica como por arqueólogos estadounidenses. Los autores Izaguirre y Ferrari exponen los conceptos que guían su acercamiento, como la configuración de paisajes y lugares, y a las modalidades sensoriales de los espacios vividos, los cuales se basan en historiadores y arqueólogos canadienses y europeos. Al igual que Gilio, el tiempo lo incorporan al considerar el tránsito y direccionalidad en la percepción física de lo sonoro. En el trabajo de Zubimendi, los aspectos sociales tratados se centran sobre espacios diferenciados por su uso actual, esto es, que son olvidados por ser rurales o lejanos de los centros urbanos. $\mathrm{El}$ aspecto temporal es usado, en su trabajo de recuperación y reconstrucción de la materialidad de los hechos, al identificar los lugares y caminos, algo semejante a los dos trabajos previos comentados. Pero también lo temporal está considerado en la evaluación de la memoria de un pasado reciente en el presente. Al considerar que el arte es articulador de las estrategias de movimiento conformando una semántica del paisaje, De Feo considera al espacio en donde tiene lugar la práctica y relación social, las cuales delinean las estrategias de configuración del paisaje. Lo temporal lo incluye en dos escalas, al trazar los senderos y su direccionalidad y al diferenciar la cronología de uso de esos caminos. Para su marco conceptual remite a arqueólogos de Chile y arqueólogos y geógrafos europeos. Para Moralejo et al., el aspecto social del espacio se refleja en el modelo social de organización inferido a partir del diseño del sitio y la logística de comunicación inka. En este caso, los autores incluyen la escala temporal al considerar la evolución del paisaje. Estos son conceptos ya empleados dentro de su equipo de investigación, por los que no elabora la red de conocimiento de referencia. Para Cahiza et al., la organización espacial consiste en procesos acumulativos en un tiempo dado de la dinámica social. Es decir que lo temporal está incluido en su concepción de lo espacial y apela a conceptos desarrollados por arqueólogos de Estados Unidos y Europa. En este aspecto, hay obvios puntos de contacto con el concepto que propone Moreno, en cuanto a que el paisaje es lo experimentado, construido o vivido y articulado por la práctica social. Pero, en este último caso, los autores a los que remite Moreno son en gran parte colegas de la Argentina, Sudamericanos y europeos.

Lo que destaco al examinar estos conceptos de territorio, espacio y tiempo es que los marcos teóricos de referencia son muy diversos, aun cuando hay aspectos comunes en el uso y aplicación de los conceptos. Si bien marqué las disciplinas y países de origen de los referentes, si se compara la bibliografía empleada en cada trabajo, resulta evidente que no hay casi ninguna coincidencia en los autores citados. Más aún, dentro del espectro de referentes, no necesariamente predominan los autores americanos o europeos y los trabajos incluyen un gran aporte de autores de Sudamérica y de la propia Argentina.

\section{Visión de conjunto}

Hay varios aspectos de este volumen que me parecen importante destacar. Para comenzar, son prueba de que el uso de las herramientas analíticas espaciales para el estudio del paisaje, en especial los SIG, pueden emplearse en cualquier etapa de la 
investigación, desde la exploración hasta la síntesis, y en cualquier escala espacial. En reuniones científicas previas, como el simposio que dio lugar a este volumen, se ha podido observar la revitalización de proyectos de largo alcance, conformando extensas bases de datos. Este es el caso del trabajo realizado en la localidad chilena de Los Vilos, por el equipo liderado por el querido Donald Jackson y Roxana Seguel (Figuerero Torres, 2015). La incorporación de esta herramienta en los diseños de investigación tampoco condiciona necesariamente su uso, siendo en realidad un gran disparador de ideas y un habilitador de otros desarrollos originales, como es el caso de la aplicación del LIDAR o de la arqueología sensorial. Por último, quisiera destacar la relevancia de lo que significa el creciente uso de referentes conceptuales regionales para los diseños de investigación locales y lo que cada trabajo ha incorporado de la producción de conocimiento regional y local. Lejos de convertirse en una investigación aislada del quehacer científico global, diseños como los expuestos en este volumen tienen el potencial de ser a la vez referentes en la producción de conocimiento regional y continental.

\section{Apreciación final}

Los trabajos de este volumen se centran en la tecnología de análisis espacial y parecen haber tomado nota del consejo de Muhammad Yunus citado al comienzo. Los casos de recolección y procesamiento de la información están fundados en usos variados de la tecnología, tanto en las aplicaciones de SIG como en las más complejas reconstrucciones para la arqueología sensorial sonora y el relevamiento con LIDAR. Pero el verdadero valor de esta colección reside en las ideas que propulsaron estos casos de estudio y en los marcos conceptuales diversos que los nutrieron. La meta de trascender fronteras y tiempos que propuso este volumen se logró con la innovación y originalidad en los resultados alcanzados que aquí se ofrecen. 


\section{Q Referencias citadas}

" Figuerero Torres, M. J. (2015). Una década de aplicaciones SIG en Arqueología Sudamericana: reflexiones y comentarios. En Actas del XIX Congreso Nacional de Arqueología Chilena (octubre 2012, Arica-Chile) (pp. 75-78). Santiago de Chile: Universidad de Tarapacá, Sociedad Chilena de Arqueología.

"Osorio Pefaur, M. (2015). La tragedia obrera de Bajo Pisagua. Río Baker, 1906. Coyhaique: Nire Negro Ediciones.

"Yunus, M. (2012, octubre 10). A Global Conversation to Join [Blog]. https://www. huffingtonpost.com/muhammad-yunus/a-global-conversation-to-_b_1951292.html 